\title{
A comparative study of high resolution transmission electron microscopy, atomic force microscopy and infrared spectroscopy for GaN thin films grown on sapphire by metalorganic chemical vapor deposition
}

\author{
Zhe Chuan Feng ${ }^{\mathrm{a}, *}, \mathrm{Kun} \mathrm{Li}^{\mathrm{b}}$, Yun Tian Hou ${ }^{\mathrm{c}, 1}$, Jie Zhao ${ }^{\mathrm{d}}$, W. Lu ${ }^{\mathrm{e}}$, W.E. Collins ${ }^{\mathrm{e}}$ \\ ${ }^{a}$ Graduate Institute of Electro-Optical Engineering and Department of Electrical Engineering, National Taiwan University, Taipei, 106-17 Taiwan, ROC \\ ${ }^{\mathrm{b}}$ Chartered Semiconductor Manufacturing Ltd., 60 Woodlands Industrial Park D, Street 2, Singapore 738406, Singapore \\ ${ }^{\mathrm{c}}$ Institute of Microelectronics, Singapore 117602, Singapore \\ ${ }^{\mathrm{d}}$ College of Physics and Electronic Information, Tianjin Normal University, Tianjin 300074, PRC \\ ${ }^{\mathrm{e}}$ Department of Physics, Fisk University, Nashville, TN 37208, USA
}

Available online 10 August 2005

\begin{abstract}
A comparative study of GaN grown on sapphire by metalorganic chemical vapor deposition (MOCVD) is performed by transmission electron microscopy (TEM), atomic force microscopy (AFM) and infrared reflectance. A correlation between the microstructure features revealed by TEM/AFM and optical characteristics obtained from infrared reflectance is explored. TEM observations reveal the GaN epilayers with high densities of threading dislocations. Dislocations in the undoped GaN tend to form open core structures, while dislocation lines in the Si-doped GaN are sharper and the strain contrast is much more discrete. Also the GaN buffer layer grown at low temperature is found to transform into the thermodynamically stable wurtzite structure during high temperature post-buffer GaN growth. The infrared reflectance shows the corresponding behavior. The interference fringes of the Si doped sample were observed with the reflectance reduction and contrast damping, which can be interpreted by the presence of a transition/defect layer near the interface of GaN/ sapphire.
\end{abstract}

(C) 2005 Elsevier B.V. All rights reserved.

Keywords: GaN; Sapphire; TEM; FTIR; Reflectance; AFM

\section{Introduction}

GaN and related III-nitride semiconductors possess large direct bandgaps, extremely high hardness, very large heterojunction offsets, high thermal conductivity and high melting temperature, and great breakthroughs have been made in recent years for their materials growth and device manufactures in applications in blue-UV light emitting diode (LED), laser diode (LD) and other optoelectronic and electronic devices [1-4]. In spite the progress achieved,

\footnotetext{
* Corresponding author. Tel.: +88623366 3543; fax: +88622367 7467 .

E-mail address: zcfeng@cc.ee.ntu.edu.tw (Z.C. Feng), ythou@tsmc.com (Y.T. Hou).

${ }^{1}$ Present address: Taiwan Semiconductor Manufacturing Corporation (TSMC), Hsinchu, Taiwan.
}

there still exist some technical barriers. One of the challenges is the lack of a suitable substrate. Sapphire is currently the most commonly used substrate. All the difficulties in hetero-epitaxy exist in the growth of $\mathrm{GaN}$ on sapphire: different chemistry $\left(\mathrm{GaN}\right.$ and $\left.\mathrm{Al}_{2} \mathrm{O}_{3}\right)$, different crystalline structure (wurtzite or sphalerite for $\mathrm{GaN}$ and corundum for sapphire), and large lattice mismatch (14\%). The resultant dislocation density in $\mathrm{GaN}$ is very high (in the order of $10^{7}-10^{9} / \mathrm{cm}^{2}$ ), even though much reduction on the dislocation density in $\mathrm{GaN}$ materials has been achieved in recent years [5]. There is a need to characterize these defects to understand the defect nature and formation mechanism, and this will facilitate the development of techniques to reduce the defect density and increase the device performance. To approach this goal, we present here a combined study, employing high resolution transmission electron 
microscopy (TEM), atomic force microscopy (AFM) and Fourier transform infrared (FTIR) spectroscopy, on the interface between $\mathrm{GaN}$ and sapphire, where most of the defects originate.

Infrared (IR) reflectance spectroscopy, a non-destructive technique, has been widely used to study the optical and structural properties of semiconductor thin films and structures $[6,7]$. It has been applied to study GaN epilayers and structures in recent years [8-13]. The electrical properties, such as carrier concentration and mobility, can be deduced from these optical measurements [9-12]. TEM is a powerful technique to study the microstructures of $\mathrm{GaN} /$ sapphire [5,14-18]. But few studies were reported to correlate the infrared reflectance data with the TEM structural observation. In this paper, efforts are made to explore the correlation between the optical IR features and microstructure characteristics revealed by TEM for the GaN/ sapphire system, which is useful to deepen our understanding of dislocation related microstructures and widen the applications of IR technology in GaN-based materials and structural devices.

\section{Experimental}

GaN thin films were grown on (0001)-plane of sapphire substrates by metalorganic chemical vapor deposition (MOCVD), using an EMCORE D180 GaN system with the vertical growth configuration and high speed rotating disk. The chemical precursors used for gallium, indium, aluminum, and nitrogen are trimethylgallium (TMG, $\mathrm{Ga}\left(\mathrm{CH}_{3}\right)_{3}$ ), trimethylindium (TMIn, $\left.\mathrm{In}\left(\mathrm{CH}_{3}\right)_{3}\right)$, trimethylalminum (TMAl, $\left.\mathrm{Al}\left(\mathrm{CH}_{3}\right)_{3}\right)$, and ammonia $\left(\mathrm{NH}_{3}, 100 \%\right.$ ), respectively. Monosilane $\left(\mathrm{SiH}_{4}\right)$ was used for n-type doping of $\mathrm{GaN}$ [10]. To overcome the negative effect of significant lattice mismatch between the $\mathrm{GaN}$ overlayer and sapphire substrate, a $200-300 \AA$ thick GaN buffer layer, amorphous or polycrystalline, was first grown on the sapphire substrate at a low temperature (LT) of $530{ }^{\circ} \mathrm{C}$. The temperature was subsequently raised to $1040{ }^{\circ} \mathrm{C}$ and the $\mathrm{GaN}$ epilayer was grown to a thickness of about $2 \mu \mathrm{m}$. Un-intentionally doped and Si-doped GaN films on sapphire were investigated in this study.

Samples for TEM cross-section measurements were prepared using the commonly adopted grinding-polishingion-milling method. First, two $2.5 \times 1.5 \mathrm{~mm}^{2}$ pieces of GaN were cut out from the sample and then glued together using G1 epoxy. The glued sample was then ground and polished from one side to a thickness of about $200 \mu \mathrm{m}$. After that, the polished sample was mounted to a $\Phi 3 \mathrm{~mm}$ copper grid, with the polished side facing done. The sample was then further ground/polished to a thickness of about $80 \mu \mathrm{m}$. Following this, dimpling was applied until the sample was about $15-\mu \mathrm{m}$ thick. Finally, the sample was ion milled in a Gatan PIPS, with the ion beam energy set at $4.5 \mathrm{kV}$ and ion milling angle at $\pm 4^{\circ}$. The prepared sample is then observed in a field emission Philips CM300 with ultra twin objective lens design.

Atomic force microscopy (AFM) was used to inspect the surface morphology of the GaN samples. Room temperature (RT) infrared spectra were measured by a Perkin Elmer FTIR spectrometer at near normal incidence with a spectral resolution of $1 \mathrm{~cm}^{-1}$.

\section{Results}

\subsection{Cross-section TEM observation}

Fig. 1 shows the bright field cross-section TEM (XTEM) images of the undoped $\mathrm{GaN}$ sample A (Fig. 1a) and Sidoped GaN sample B (Fig. 1b). Both images show dislocation lines from the interface to the GaN film surface with high dislocation densities: the threading dislocation density in undoped $\mathrm{GaN}$ is about $9 \times 10^{8} / \mathrm{cm}^{2}$, while the threading dislocation density in Si-doped $\mathrm{GaN}$ is about $6 \times 10^{8} / \mathrm{cm}^{2}$.
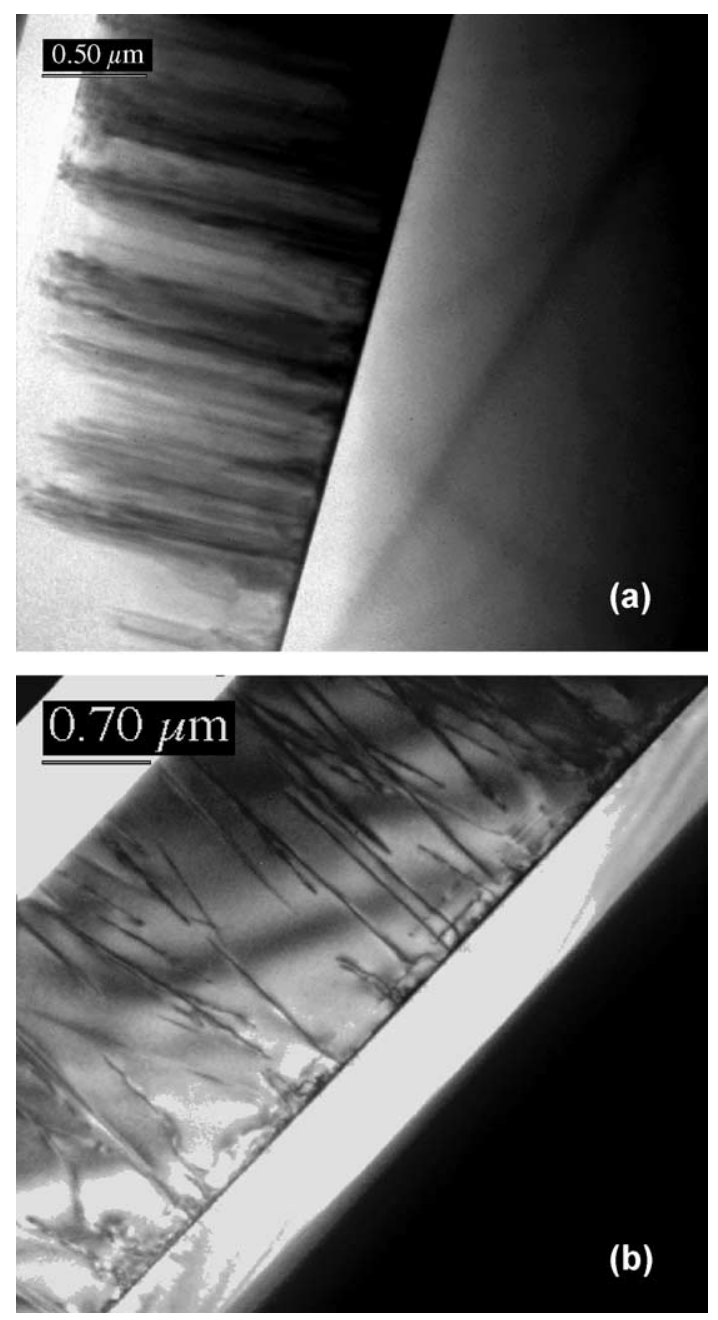

Fig. 1. Cross-section TEM images of two MOCVD samples; (a) undoped GaN and (b) Si-doped GaN. 


\subsection{AFM surface morphology}

Fig. 2 shows the AFM observation of the film surface morphology, where the holes at the surface can be clearly seen. There are more holes at the surface of undoped $\mathrm{GaN}$ than that of Si-doped GaN, and the holes at the surface of undoped $\mathrm{GaN}$ are on average larger than the holes at the $\mathrm{Si}$ doped GaN surface.

\subsection{High resolution TEM imaging}

The high resolution TEM images of the $\mathrm{GaN} / \mathrm{Al}_{2} \mathrm{O}_{3}$ interface for these two samples are shown in Fig. 3. In Fig. 3(a) for the undoped $\mathrm{GaN}$ sample, the sapphire region is shown in the right-low side large area, and the GaN film region is seen on the left-up corner, while the middle declined area is represented for the transition buffer layer region. For the Si-doped $\mathrm{GaN} /$ sapphire sample as shown in Fig. 3(b), the sapphire region is on the left-up corner, and the GaN film region is on the right-down area, while the middle declined area is represented for the transition buffer layer region too. Similar to Fig. 1, the undoped GaN shows more obvious strain contrast, especially in the buffer layer region, than the Si-doped GaN.
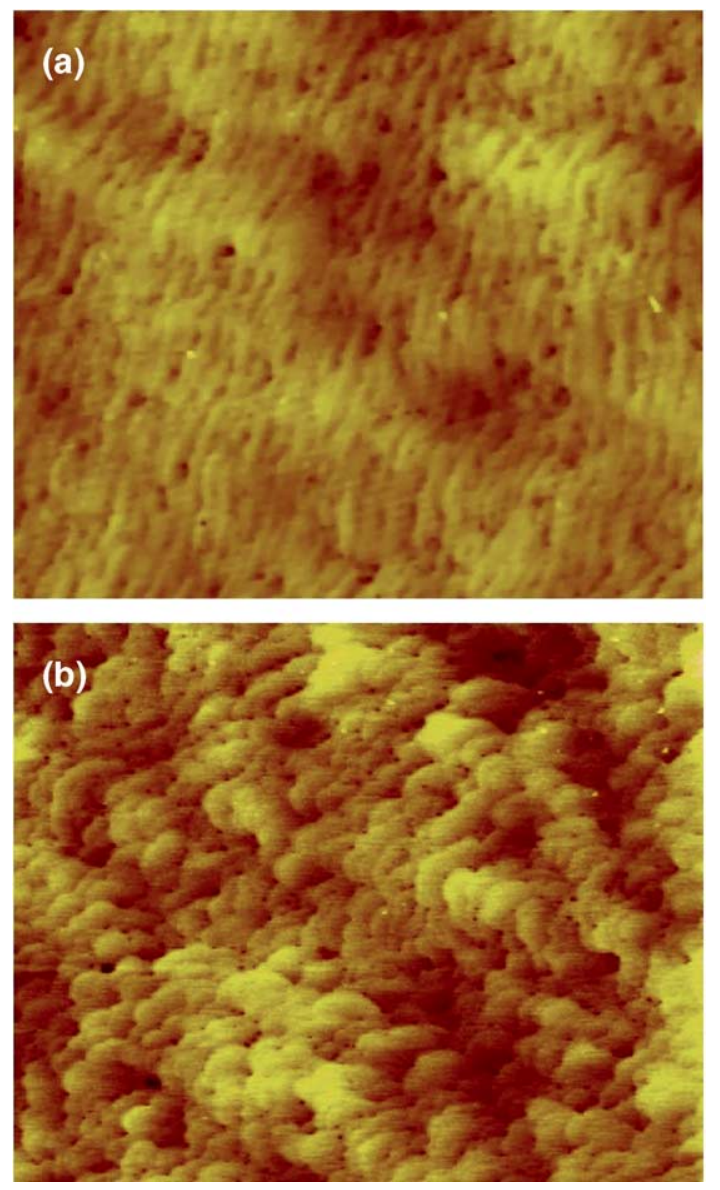

Fig. 2. AFM images of (a) undoped GaN and (b) Si-doped GaN.
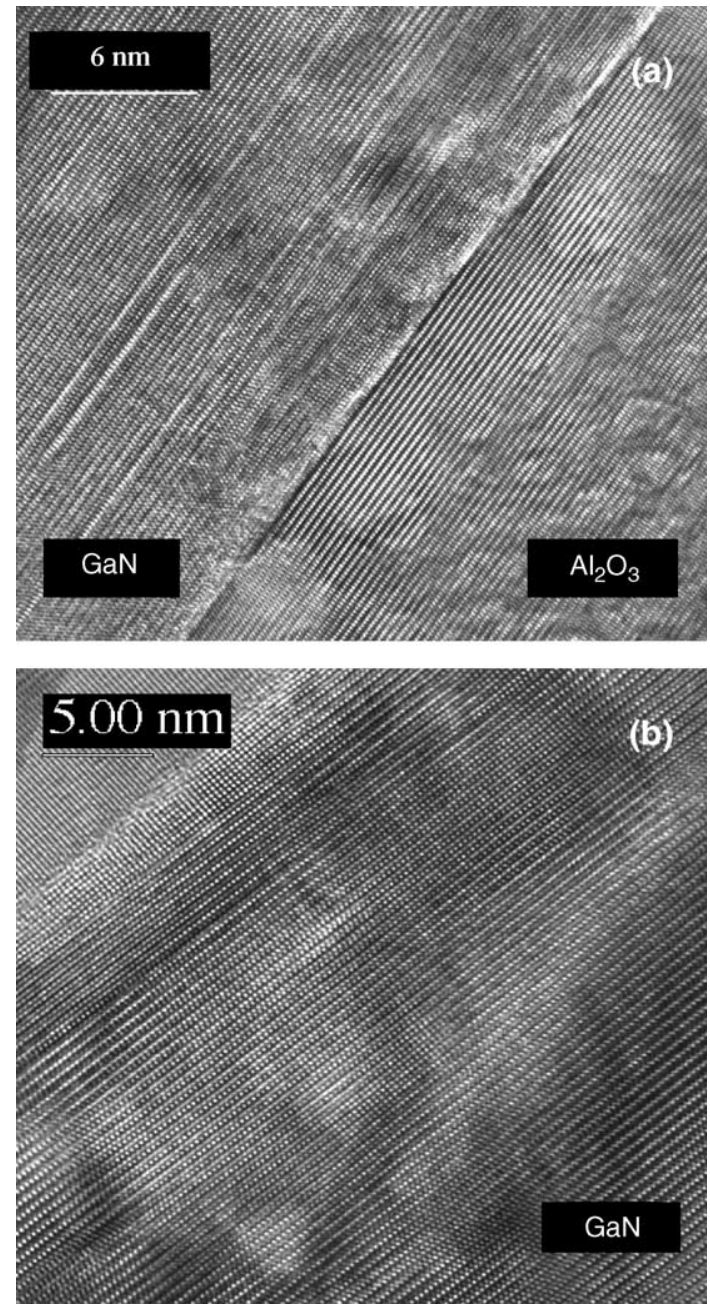

Fig. 3. High resolution TEM images of (a) undoped GaN and (b) Si-doped $\mathrm{GaN}$.

\subsection{Electron diffraction pattern}

A typical corresponding electron diffraction pattern is shown in Fig. 4. Two sets of patterns from GaN and

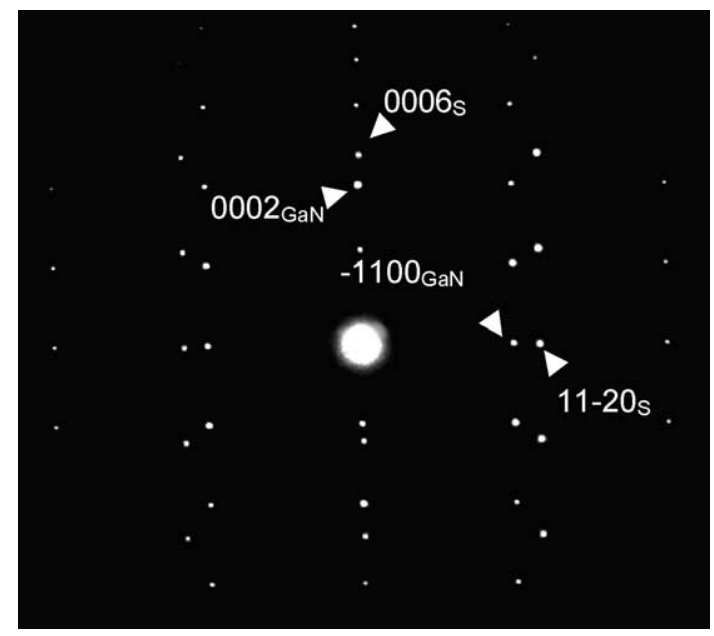

Fig. 4. Electron diffraction pattern from $\mathrm{GaN} / \mathrm{sapphire}$ interface. 
sapphire respectively can be easily distinguished and indicated with subscript "GaN" for patterns representative for GaN crystal and "s" for sapphire. More description will be given in the discussion section.

\subsection{FTIR reflectance and primary simulation}

Fig. 5 shows IR reflectance spectra of two typical undoped and Si-doped GaN/sapphire with solid lines representing experimental data. The theoretical primary simulation on IR spectra has been done, based upon a single oscillator model $[6,8,10]$ :

$\varepsilon=\varepsilon_{\infty}\left(1+\frac{\omega_{\mathrm{LO}}^{2}-\omega_{\mathrm{TO}}^{2}}{\omega_{\mathrm{TO}}^{2}-\omega^{2}-i \gamma_{\mathrm{TO}} \omega}-\frac{\omega_{\mathrm{p}}^{2}}{\omega\left(\omega+i \gamma_{\mathrm{p}}\right)}\right)$

where the second term is the dispersion due to phonons and the third term arises from free carriers. Here $\varepsilon_{\infty}$ is the high frequency dielectric constant, $\omega_{\mathrm{LO}}$ and $\omega_{\mathrm{TO}}$ are the LO and TO phonon frequency, $\gamma_{\text {TO }}$ is the damping factor of the transverse optic modes, $\omega_{\mathrm{p}}$ is the plasma frequency and $\gamma_{\mathrm{p}}$ is the electron damping constant, respectively. Fig. 5(a) and (b1) show the essential simulations using Eq. (1) in dotted lines for the two samples, with parameters of $\varepsilon_{\infty}=5.2$, $\omega_{\mathrm{LO}}=742 \mathrm{~cm}^{-1}, \omega_{\mathrm{TO}}=560 \mathrm{~cm}^{-1}$ and $\gamma_{\mathrm{TO}}=5 \mathrm{~cm}^{-1}$ for
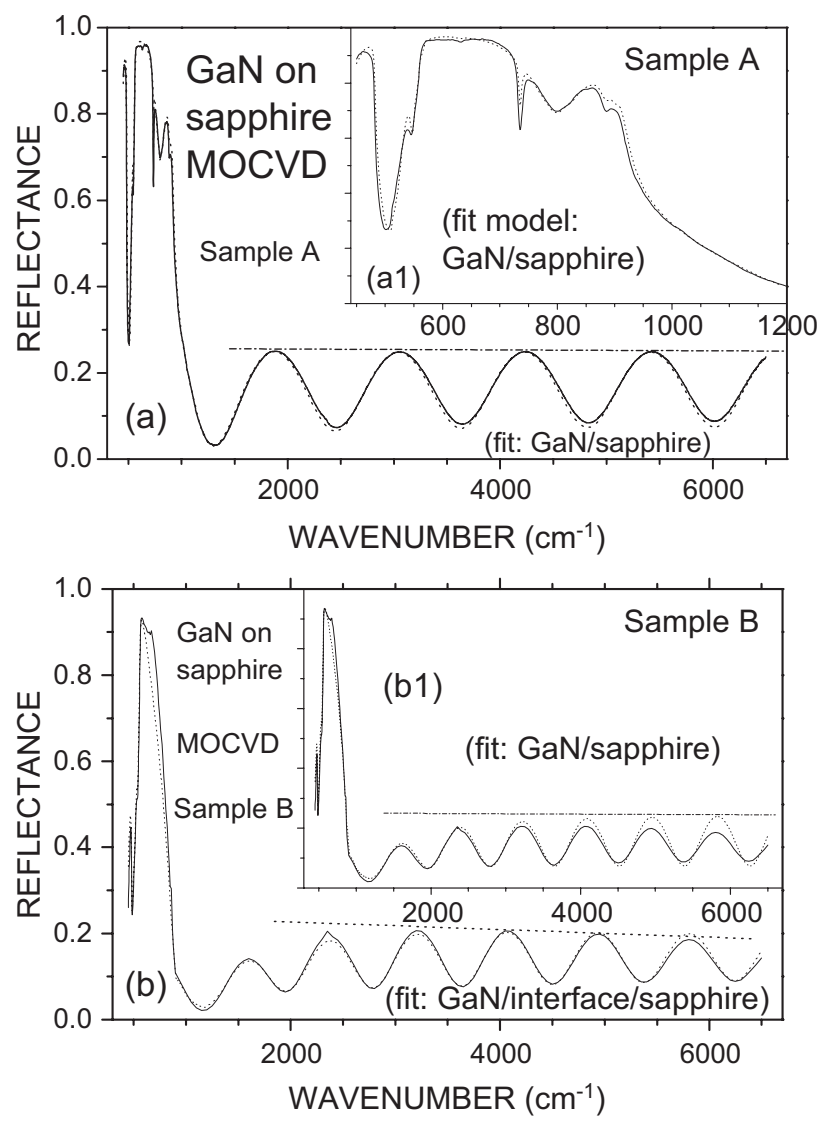

Fig. 5. Infrared reflectance of two samples of MOCVD-grown GaN/ sapphire samples, with solid lines for experiment data and dotted/dash-dot lines for simulation results. both samples, $\omega_{\mathrm{p}}=0$ and $\gamma_{\mathrm{p}}=0$ for sample $\mathrm{A}$, and $\omega_{\mathrm{p}}=700$ $\mathrm{cm}^{-1}$ and $\gamma_{\mathrm{p}}=400 \mathrm{~cm}^{-1}$ for sample $\mathrm{B}$, respectively.

\section{Discussion}

\subsection{Dislocation features from TEM and AFM observation}

As revealed from Fig. 1, the dislocation structures are obviously different from the undoped and Si-doped GaN/ sapphire samples. In Si-doped $\mathrm{GaN}$, the strain contrast caused by threading dislocations is more confined and sharp dislocation lines can be clearly seen; while in the undoped $\mathrm{GaN}$, the strain field caused by threading dislocations is much broader, and the dislocations tend to form open core structures.

The AFM image of the surface for the undoped GaN in Fig. 2(a) shows the well-defined steps and terraces, which is evidenced for the two-dimensional step-growth [19]. The step height of $0.3 \mathrm{~nm}$ corresponds to one monolayer of $(0001)_{\mathrm{h}} \mathrm{GaN}$ along the $c$-axis $(c / 2=0.26 \mathrm{~nm})$. The dark spots on the image correspond to step terminations. These step terminations on a single crystal surface must represent the intersection of threading dislocations with the free surface. The threading dislocations observed in X-TEM must have either pure screw or mixed screw-edge character (a pure edge threading dislocation will not lead to a step termination) [19].

In the AFM image of Fig. 2(b) for the heavily Si-doped GaN surface, the step-terrace features are not so obvious as for the undoped $\mathrm{GaN}$ in Fig. 2(a). But the dark pits and holes can be seen clearly. There are more holes at the surface of undoped GaN than that of Si-doped $\mathrm{GaN}$, and the holes at the surface of undoped GaN are on average larger than the holes at the Si-doped GaN surface. These features are due to the influence from the heavy Si-dopants into the $\mathrm{GaN}$ thin films, which modified the surface of GaN crystal.

\subsection{Frank dislocation loops}

The dislocations that were observed from XTEM in Fig. 1 and revealed with terminations from AFM in Fig. 2 tend to form open core structures. According to Frank's energy competition theory $[5,20]$, whether an open core dislocation structure will be formed or not depends on the balance between the strain energy of a dislocation core and the extra surface energy when an open core structure is formed. In other words, an open core dislocation will be formed if there is a decrease in the total energy by replacing the highly strained dislocation core with an empty pipe. The essence of this theory is that the open core dislocation structure is surface energy related. We have already found in our AFM study that different dopants can cause significant change in the surface morphology of GaN. It is well known that different surface morphologies are related to different growth modes, which is a result of different surface energy. 
Therefore, it is believed that Si doping causes the increase in surface energy of $\mathrm{GaN}$, which results in a transformation from open core dislocation pipes to more confined dislocation lines. It is also noted that in Fig. 1(b) there are Frank dislocation loops located at a distance of about $110 \pm 10 \mathrm{~nm}$ from the interface, and the loops seem to form a secondary 'interface'.

\subsection{Stacking sequence of the atomic planes revealed from high resolution TEM}

As found from the high resolution TEM images of the $\mathrm{GaN} / \mathrm{Al}_{2} \mathrm{O}_{3}$ interface for two samples in Fig. 3, the undoped $\mathrm{GaN}$ shows more obvious strain contrast, especially in the buffer layer region, than the Si-doped GaN. Careful examination on the stacking sequence of the atomic planes along the (0001) direction reveals that the thermodynamically unstable sphalerite structure formed during low temperature $\left(530^{\circ} \mathrm{C}\right)$ buffer layer growth has transformed into the thermodynamically stable wurtzite structure after high temperature $\left(1030{ }^{\circ} \mathrm{C}\right)$ epilayer growth. The stacking sequence of (0001) planes in the buffer layer is $\cdots$ ABABAB $\cdots$ for wurtzite, rather than $\cdots A B C A B C \cdots$ for sphalerite in both undoped and Si-doped GaN. Compared with the buffer layer, the epilayer crystallinity is much better; and there are fewer stacking faults in the epilayer. Two types of common stacking faults, extrinsic and intrinsic, can be found in the buffer layer region. The extrinsic stacking fault is formed through the insertion of half an atomic (0001) plane, where the stacking sequences changes from $\cdots$ BABABABA $\cdots$ in perfect region to $\cdots B A B A C B C B \cdots$ in the faulted region, while the intrinsic stacking fault is formed through the extraction of half an atomic (0001) plane, where the stacking sequences changes from $\cdots$ BABABABA $\cdots$ in perfect region to "BABACACA $\cdots$ in the faulted region.

\subsection{Electron diffraction pattern}

From the electron diffraction pattern in Fig. 4, two sets of patterns from $\mathrm{GaN}$ and sapphire respectively are clearly exposed. Since sapphire possesses a smaller lattice parameter than $\mathrm{GaN}$, the spacing of its diffraction pattern in reciprocal space will be larger as compared with that of $\mathrm{GaN}$. From the indexing of this diffraction pattern, it is clear that the electron beam is coming down along the [1120] direction of $\mathrm{GaN}$ or [1100] direction of sapphire. The orientation relationship between $\mathrm{GaN}$ and sapphire can also easily defined as $[11 \overline{2} 0]_{\mathrm{GaN}} / /[1 \overline{1} 00]_{\mathrm{sapphire}}$, and $[\overline{1} 100]_{\mathrm{GaN}} / /$ $[11 \overline{2} 0]_{\text {sapphire. }}$

In previous TEM studies, Ruvimov et al. [15] reported the effect of Si doping on the dislocation structure of GaN/ sapphire, showing the decrease of dislocation density and the change of dislocation arrangement toward a more random distribution, and Xin et al. [16] reported the observation of the core structures of threading dislocation in GaN. Our investigation above has further deepened the physical understanding for the dislocation structures in $\mathrm{GaN} / \mathrm{sapphire}$ from the surface energy analysis. Furthermore, these microstructural features can be correlated with optical properties from FTIR measurements, discussed in next section.

\subsection{Detailed features revealed from FTIR reflectance spectroscopy}

From the primary theoretical simulation fits for FTIR reflectance of $\mathrm{GaN} /$ sapphire in Section 3.1, the carrier concentration $n$ can be obtained via the relationship:

$\omega_{\mathrm{p}}^{2}=\frac{n e^{2}}{m^{*} \varepsilon_{\infty} \varepsilon_{0}}$

where the perpendicular effective mass of electrons in $\mathrm{GaN}$, $m^{*}$, is $0.22 m_{0}$ [21], and $m_{0}$ is the free electron mass. For sample B, fitting to the IR reflectance below $1200 \mathrm{~cm}^{-1}$ leads to a carrier concentration of $6.3 \times 10^{18} \mathrm{~cm}^{-3}$, which is consistent with Hall measurements $\left(6.2 \times 10^{18} \mathrm{~cm}^{-3}\right)$.

It can also be seen that excellent agreement between experimental and simulation data is obtained for the high quality undoped GaN sample A [Fig. 5(a)]. However, there exist some deviations for the heavily Si-doped sample B using the same simulation method [Fig. 5(b1)]. This difference is indeed correlated with micro-structural differences, as observed by TEM and AFM, discussed above.

Let's further examine the IR experimental and simulation results. For sample A, it shows from Fig. 5(a) and (a1) that the simulation agrees well with the experiment for the whole spectral region. Fig. 5(a1) expands in details the reflectance data and fits between 400 and $1200 \mathrm{~cm}^{-1}$, i.e. in the so-call "reststrahlen" $[6,10]$ band region, where optical reflectance is caused from lattice phonon vibrations. Between two phonon frequencies of transverse optical (TO) and longitudinal optical (LO) phonons, the reflectance is shaped as a "flat top" with near $100 \%$ reflectivity $(96 \%$ here). These indicate the good crystalline quality of the film. The successful application of the simple film/substrate model also demonstrates that the interface of film and substrate is abrupt, in agreement with our TEM observations.

However, the "flat top" is declined for the case of sample B. Beyond $1200 \mathrm{~cm}^{-1}$ in Fig. 5(b1), the calculation shows significant deviation from the experiment data for the interference fringes, which exhibit an intensity dependence on photon frequency in contrast with the case of the high quality undoped GaN/sapphire in Fig. 5(a).

We have examined more than 50 samples, and found that all high quality undoped films possess IR reflectance similar to that in Fig. 5(a) and can be well fitted with Eq. (1). The interference patterns, i.e. Fabry-Perot oscillations beyond $1200 \mathrm{~cm}^{-1}$ (data and fits), are always with equal intervals for maxima and minima, and with equal reflectivity for all maxima [see the dot line in Fig. 5(a)]. For heavily doped GaN/sapphire samples, the experimental IR reflectance 
always show a damping behavior, i.e. the intensity at maxima decreases with frequency (also accompanying a declining of the reflectance between TO and LO mode frequencies). Their theoretical fits using the simple GaN/ sapphire model can only lead to oscillation curves without any damping (no matter how to change the parameters) as shown in Fig. 5(b1).

To approach good simulation fits for the heavily doped GaN samples, we propose the presence of an interface layer between the substrate and epitaxial layer. In the calculation, the interface layer is considered simply as a mixture of $\mathrm{GaN}$ and voids, interpreted as an effective optical transition layer induced by imperfections in GaN near the interface.

It is also observed in our TEM study in Fig. 1 (b), sample B has many Frank dislocation loops lined up at a distance of about $110 \pm 10 \mathrm{~nm}$ from the $\mathrm{GaN} / \mathrm{sapphire}$ interface, and the loops seem to form a secondary 'interface'. Since Frank dislocation loops are formed through the collapse of voids, the above void/GaN assumption is reasonable. After taking this assumption into the theoretical model and noting that the refractive index of $\mathrm{GaN}$ is constant in regions away from the lattice phonon modes, a good fit with data is achieved as shown in Fig. 5(b). The GaN/sapphire interface characteristics can be obtained from the damping behavior of the interference fringe contrast. The percentage of voids in the interface layer is $15 \%$ and the thickness of the interface layer obtained for sample B is $0.09 \mu \mathrm{m}$, which is quite close to the depth of Frank dislocation loops observed in TEM.

\section{Conclusion}

In conclusion, the influence of dopants on the dislocation structure and its further influence on the IR response of GaN films are studied by cross-section and high resolution TEM, AFM and FTIR. Comparative TEM and FTIR study shows that both the undoped and Si-doped GaN have good film quality and abrupt GaN/sapphire interfaces. Dislocations in the undoped $\mathrm{GaN}$ tend to form open core structures with the terminations revealed from AFM pattern, while dislocation lines in the Si-doped GaN are very sharp and the strain contrast is much more confined. Based upon the energy competition theory and analysis, it demonstrates that $\mathrm{Si}$ doping causes the increase in surface energy, resulting in the change in dislocation configuration from open core structures in undoped $\mathrm{GaN}$ to much more confined dislocation lines. In the Si-doped GaN, nearly continuous Frank dislocation loops are formed at a depth of about $110 \pm 10 \mathrm{~nm}$ from the GaN/ sapphire interface, and these Frank dislocation loops parallel to the interface, are responsible for the damping and reflection reduction in the IR spectra of the Si-doped GaN. The corresponding model with the presence of a transition/ defect layer near the interface and its simulation account well for the observed IR spectrum. A correlation between the optical characteristics from IR reflectance spectroscopy and microstructure features revealed by TEM and AFM for
MOCVD grown GaN/sapphire is established. This work is not only significant in academy but also useful in industry production for $\mathrm{GaN}$ materials.

\section{Acknowledgements}

We acknowledge the help and support from Profs. A.T.S. Wee, J. Lin, S.J. Chua and C.C. Yang. The work at National Taiwan University was supported by funds from National Science Council of Republic of China, NSC 93-2218-E002-011 and 93-2215-E-002-035.

\section{References}

[1] S. Nakamura, S. Pearton, G. Fasol, The Blue Laser Diode-The Complete Story, Springer, Berlin, 2000.

[2] H. Morkoc, Nitride Semiconductors and Devices, Springer, Berlin, 1999.

[3] E.T. Yu, M.O. Manasreh (Eds.), III-V Nitride Semiconductors: Applications and Devices, vol. 16, in: M.O. Manasreh (Ed.), Optoelectronic Properties of Semiconductors and Superlattices, Taylor \& Francis, New York, 2003.

[4] M.O. Manasreh, I.T. Ferguson (Eds.), III-V Nitride Semiconductors: Growth, vol. 19, in: M.O. Manasreh (Ed.), Optoelectronic Properties of Semiconductors and Superlattices, New York, Taylor \& Francis, 2003.

[5] X.H. Wu, P. Fini, E.J. Tarsa, B. Heying, S. Keller, U.K. Mishra, S.P. Denbaars, J.S. Speck, J. Cryst. Growth 189/190 (1998) 231.

[6] S. Perkowitz, Optical Characterization of Semiconductors, Academic, San Diego, 1993, Chapter 4.

[7] G. Bauer, W. Ritcher (Eds.), Optical Characterization of Epitaxial Semiconductor Layers, Springer, Berlin, 1996, Chapter 5.

[8] G. Yu, H. Ishikawa, M. Umeno, T. Egawa, J. Watanabe, T. Soga, T. Jimbo, Appl. Phys. Lett. 73 (1998) 1472.

[9] P. Wisniewski, W. Knap, J.P. Malzac, J. Camassel, M.D. Bremser, R.F. Davis, T. Suski, Appl. Phys. Lett. 73 (1998) 1760.

[10] Y.T. Hou, Z.C. Feng, S.J. Chua, M.F. Li, N. Akutsu, K. Matsumoto, Appl. Phys. Lett. 75 (1999) 3117.

[11] J.A. Bardwell, M.W.C. Dharma-wardana, H. Tang, J.B. Webb, J. Vac. Sci. Technol., A, Vac. Surf. Films 18 (2000) 643.

[12] E. Frayssinet, W. Knap, P. Prystawko, M. Leszczynski, I. Grezegory, T. Suski, B. Beaumont, P. Gibart, J. Cryst. Growth 218 (2000) 161.

[13] X. Zhang, Y.T. Hou, Z.C. Feng, J. Chen, J. Appl. Phys. 89 (2001) 6165.

[14] F.A. Ponce, in: S.J. Pearton (Ed.), GaN and Related Materials, Gordon and Breach, Australia, 1996.

[15] S. Ruvimov, Z. Liliental-Weber, T. Suski, J.W. Ager III, J. Washburn, J. Krueger, C. Kisielowski, E.R. Weber, H. Amano, I. Akasaki, Appl. Phys. Lett. 69 (1996) 990.

[16] Y. Xin, S.J. Pennycook, N.D. Browning, P.D. Nellist, S. Sivananthan, F. Omnes, B. Beaumont, J.P. Faurie, P. Gibart, Appl. Phys. Lett. 72 (1998) 2680.

[17] D.M. Follstaedt, N.A. Missert, D.D. Koleske, C.C. Mitchell, K.C. Cross, Appl. Phys. Lett. 83 (2003) 4797.

[18] X.L. Fang, Y.Q. Wang, H. Meidia, S. Mahajan, Appl. Phys. Lett. 84 (2004) 484.

[19] D. Kapolnek, X.H. Wu, B. Heying, S. Keller, B.P. Keller, U.K. Mishra, S.P. DenBaars, J.S. Speck, Appl. Phys. Lett. 67 (1995) 1541.

[20] F.C. Frank, Acta Crystallogr. 4 (1951) 497.

[21] P. Perlin, E. Litwin-Staszewska, B. Suchanek, W. Knap, J. Camassel, T. Suski, R. Piotrzkowski, I. Grzegory, S. Porowski, E. Kaminska, J.C. Chervin, Appl. Phys. Lett. 68 (1996) 1114. 